\title{
Experimental and Modeling Studies of Engine Rubber Bushing Subjected to Impact Loading
}

\author{
Xiong-Li-Ren JIANG ${ }^{1, a,}$, Na YANG ${ }^{1, b, *}$, Tao LIU ${ }^{1, c}$ and Yao-Wei HU ${ }^{2, d}$ \\ ${ }^{1}$ School of Automotive Engineering, Harbin Institute of Technology, Weihai 264209, Shandong, \\ China \\ ${ }^{2}$ FAW-Volkswagen Automotive Co. Ltd, Changchun 130000, Jilin, China \\ ajiangxoinglr@126.com, bynhelen@163.com, 'Lt4325@163.com, dyaowei.hu@faw-vw.com
}

*Na YANG

Keywords: Rubber bushing, Shock, Experimental test, Simulation.

\begin{abstract}
In order to study the response behavior of engine rubber bushing under high speed impact, improve the simulation accuracy of the whole vehicle, the mechanical properties of rubber bushing was studied. The method of the drop-hammer impact on the rubber bushing was analyzed. A station was built with the specific shock wave method and the regulation velocity method of drop-hammer impact testing machine. The difference between the two methods in the study of rubber bushing test was compared. The rubber bushing was analyzed by finite element Simulation, analyzed with the rubber bushing experiment, the response behavior was predicted of the rubber bushing under complex operating conditions, and a general method for solving the impact characteristic of rubber bushing was analyzed.
\end{abstract}

CLC: U465.4+2 Document Code: A

\section{Introduction}

The researches of vehicle body structure are the most important part of passive safety research, uses the finite element method to mesh the vehicle body model, are more accurate model of the response of the various sub components and their connection to the real movement and mechanical properties. The solid model is solved by using the computer's powerful computing ability. The finite element method is widely used in vehicle crash simulation [1].

The numerical simulation is widely used in the passive safety performance of the automotive, and save a lot of time and money cost. However, when the finite element method is used to analyze the passive safety performance of the vehicle, a number of tests are still needed. The simulation results are modified and verified by the test results.

This paper focuses on the simulation of automotive passive safety. A test process for the study of the large deformation characteristics under the impact load of the automobile engine is set up. The method of solid modeling for engine rubber bushing is analyzed. It is verified that the simulation of the rubber bushing is used for solid modeling.

\section{Mechanical Property of the Rubber Bushing}

The ideal mechanical property of rubber material is between the ideal elastic solid model and the ideal viscous liquid model. This mechanical property is called viscoelasticity. The typical model of viscoelasticity is: Maxwell model and Voigt model. They are all based on the phenomenological constitutive model, and can be used to describe the nonlinear viscoelastic and elastic properties of ideal rubber materials [2,3].

Maxwell model is composed of ideal elastomer and ideal viscous [4]. Voigt model is composed 
of ideal elastic and the ideal viscous[5], and are shown in Figure 1.
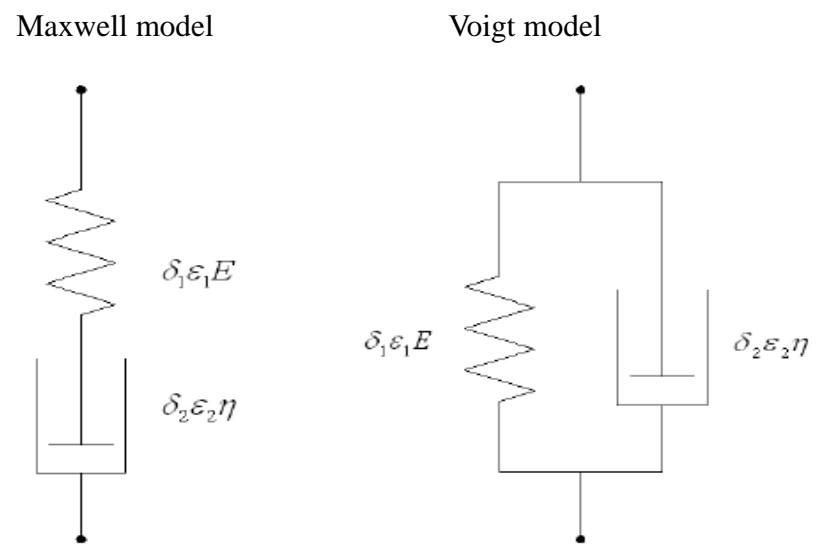

Fig.1. Maxwell model and Voigt model

\section{Design of Rubber Bushing Test Bench and Measurement System}

The test method for impact dynamic characteristics of rubber bushing both domestic and foreign, include drop method, pendulum method etc. According to the domestic situation as well as the research object, usually used drop method in the automotive field[6,7].

\section{Drop Hammer Impact Test Method}

Drop test method is divided into two types: prescribed pulse waveform method and prescribed impact velocity method. The difference between these two methods is the impact of input in different ways. Prescribed pulse waveform method uses waveform transmitter, therefore, the complexity of prescribed pulse waveform method in the test of the adjustment to more than prescribed impact velocity method.

Prescribed pulse waveform method between the base and the adapter plate mounting bushing placed waveform transmitter. The waveform transmitter is adjusted the cone angle and the height, to meet the requirements of the input pulse waveform as shown in Figure 2.

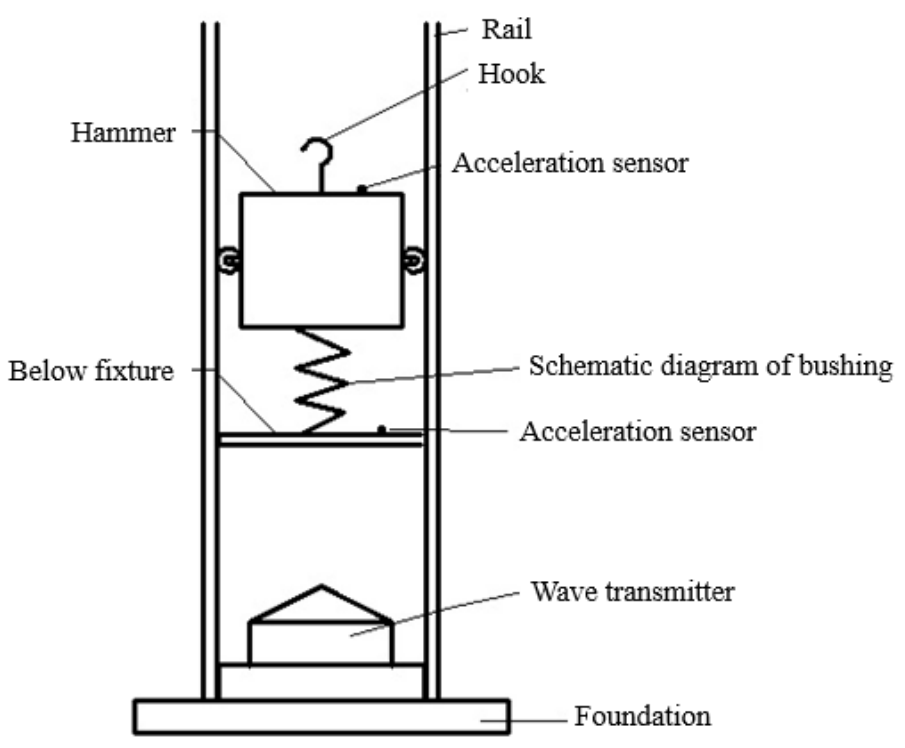

Fig.2. Schematic diagram of the experimental arrangement 
Prescribed impact velocity method is currently the most used test method, test equipment simple adjustment, high stability data, strong anti-interference. Prescribed impact velocity method has some difference from the reality.

\section{Brief introduction of the Drop Hammer Test Bench System}

A simple drop hammer impact testing machine was improved, to meet the needs of large deformation test of rubber bushing. Experimental study on a passenger car engine rubber bushing was carried out, used the improved machine.

A simple drop hammer impact testing machine is mainly composed of a drop hammer, steel rails, lifting device, a support frame, etc[8]. Drop hammer is a $50 \mathrm{~cm} \times 44 \mathrm{~cm} \mathrm{x} 12 \mathrm{~cm}$ alloy tool steel, the total mass is $205 \mathrm{KG}$.

\section{Rubber Bushing Impact Test and Data Processing}

A vehicle body acceleration process was analyzed. The acceleration can be extracted, when the engine moves forward and the rubber bushing hinders the forward movement of the engine. Acceleration waveform of the body is roughly half sine wave, the peak is about $20 \mathrm{~g}$ and the pulse width is about $10-20 \mathrm{~ms}$.

\section{Prescribed Pulse Waveform Impact Test}

After the analysis of environmental impact of rubber bushing bearing in vehicle frontal impact wave, it can be in accordance with the prescribed pulse waveform method to design waveform transmitter, setting the hammer height and other parameters, then testing the rubber bushing impact property.

\section{Test Procedure}

(1) Using drop hammer to prescribed pulse waveform test. The waveform transmitter placed just below the hammer, then use the clamp through the screw to connect the bushing to the hammer below. And as far as possible to ensure the rubber bushing center, hammer center and waveform transmitter on the center line.

(2) Drop hammer is lifted to a certain height of motor. When the pulse width is $20 \mathrm{~ms}$ and the peak value is $20 \mathrm{~g}$, the impact velocity obtained by integration, and the impact velocity is $2.543 \mathrm{~m} / \mathrm{s}$, the drop height is $0.33 \mathrm{~m}$.

(3) Install and debug the measurement system and test. According to the collected acceleration waveform, adjusted of hammer height, drop hammer mass, etc. to achieve the specified waveform. Considered the test adjustment very difficult, therefore, it needed to select the reliable test data to analysis and research.

(4) The stiffness characteristic of rubber bushing impact was analyzed.

\section{The data Acquisition and Treatment}

In accordance with the above steps, the reliable rubber bushing input waveform was collected and carefully selected, then used CFC180 on input signal filtering as shown in Figure 3.

In the figure, its pulse width is $14.7 \mathrm{~ms}$, and peak value is $19.2 \mathrm{~g}$. Compared with the standard sine wave, it is found that the difference between the input waveform and the standard sine wave is $26.5 \%$, and the difference of the peak value is $4 \%$, the error is within acceptable limits.

The output waveform collected at the center of the upper-fixture is shown in Figure 4. 


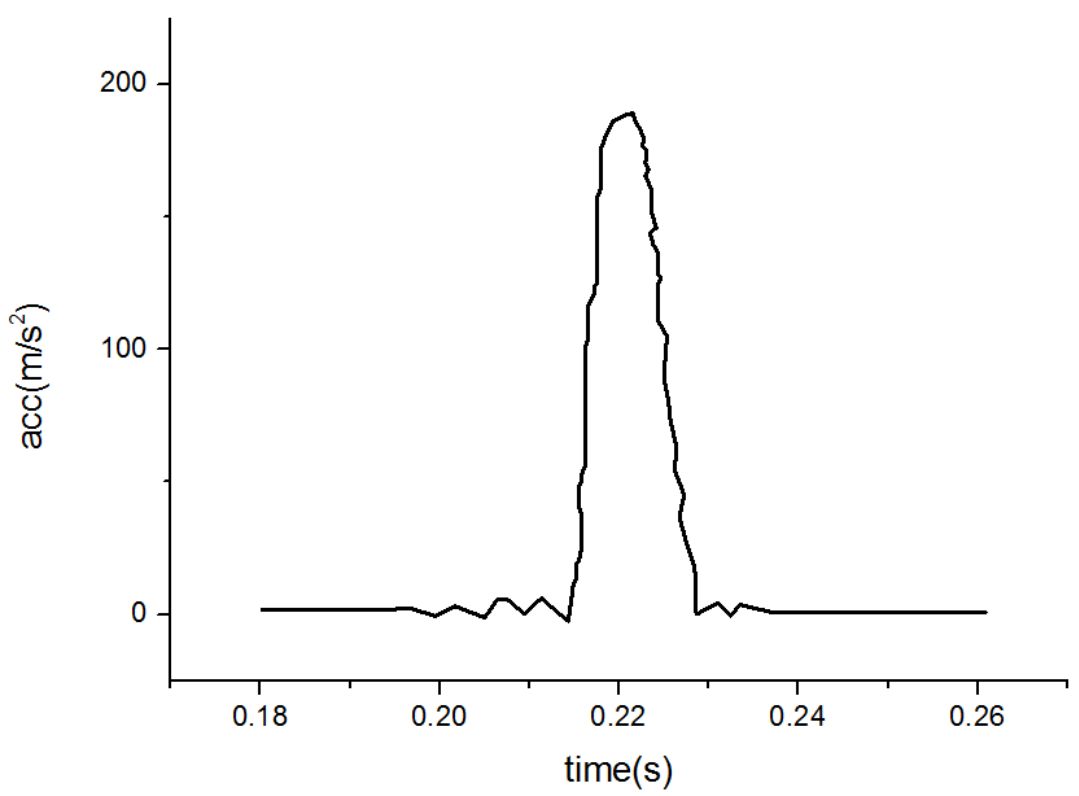

Fig.3. The input waveform from below-fixture

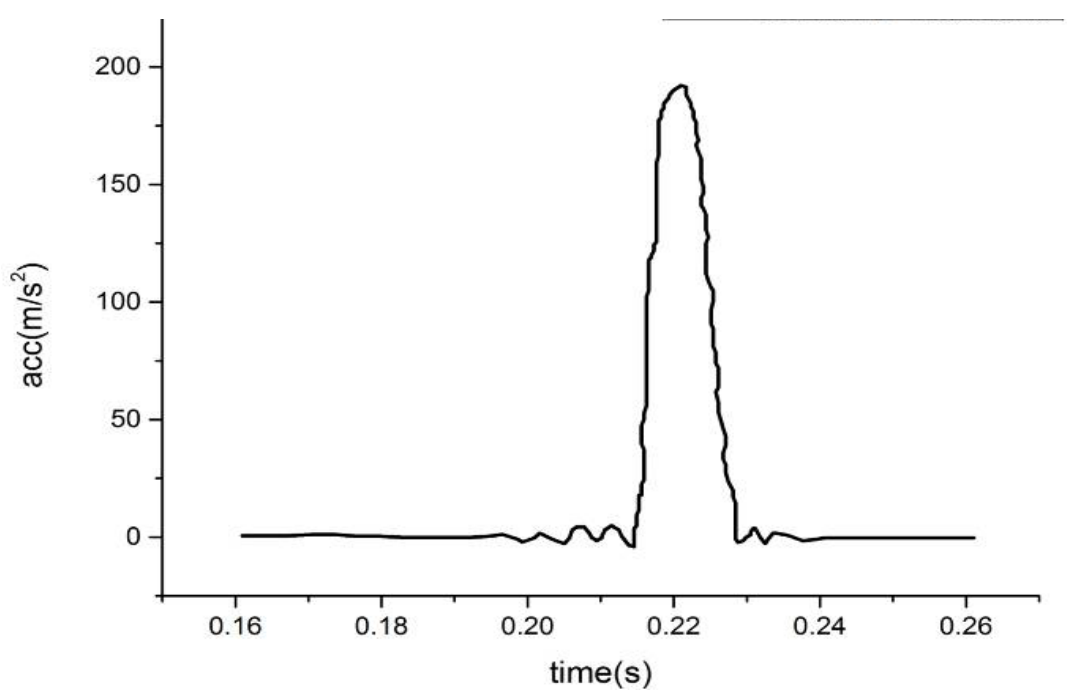

Fig.4. The input waveform from upper-fixture

Comparing the test curves, rubber bushing has hysteresis effect between the input waveform and the output waveform. This is consistent with the viscoelastic mechanical properties of rubber.

\section{Prescribed Impact Velocity Impact Test}

According to the integration of the engine wall acceleration time, the impact speed is about $2.52 \mathrm{~m} / \mathrm{s}$.

\section{Test Procedure}

(1) Using drop hammer to prescribed impact velocity test. The bushing equipment was connected with the upper-fixture and the below-fixture, and under the drop hammer and placed in the center.

(2) Drop hammer is lifted to a certain height $0.57 \mathrm{~m}$ of motor. The height is the height of the center of mass, but in the actual test, it is not easy to measure the height of the center of mass. Considering the hammer thickness is $120 \mathrm{~mm}$, and center of mass is at half the thickness of the hammer, therefore, in the actual test, it can be used under the impact hammer surface distance bushing center height is $0.51 \mathrm{M}$ to drop height.

(3) Debug the measurement system and test. The acceleration of the bushing center shaft need to collect. And the system does not require additional debug, only need to ensure consistency of 
hammer height measurement.

(4) The data integration used Origin. the impact characteristics of rubber bushing by prescribed impact velocity method was analyzed.

\section{The Data Acquisition and Treatment}

In accordance with the above steps, the acceleration waveform of the center shaft of the rubber bushing is collected, then used CFC180 on input signal filtering as shown in Figure 5 . The acceleration in the figure is integrated, the speed of the impact of the bushing is $2.71 \mathrm{~m} / \mathrm{s}$, the difference between the actual velocity and the expected impact velocity is $7.96 \%$.

Acceleration at the center of the bushing is integrated two times, obtained the displacement of the bushing center. The impact characteristic curve of the rubber bushing under prescribed impact velocity can be obtained as shown in Figure 6.

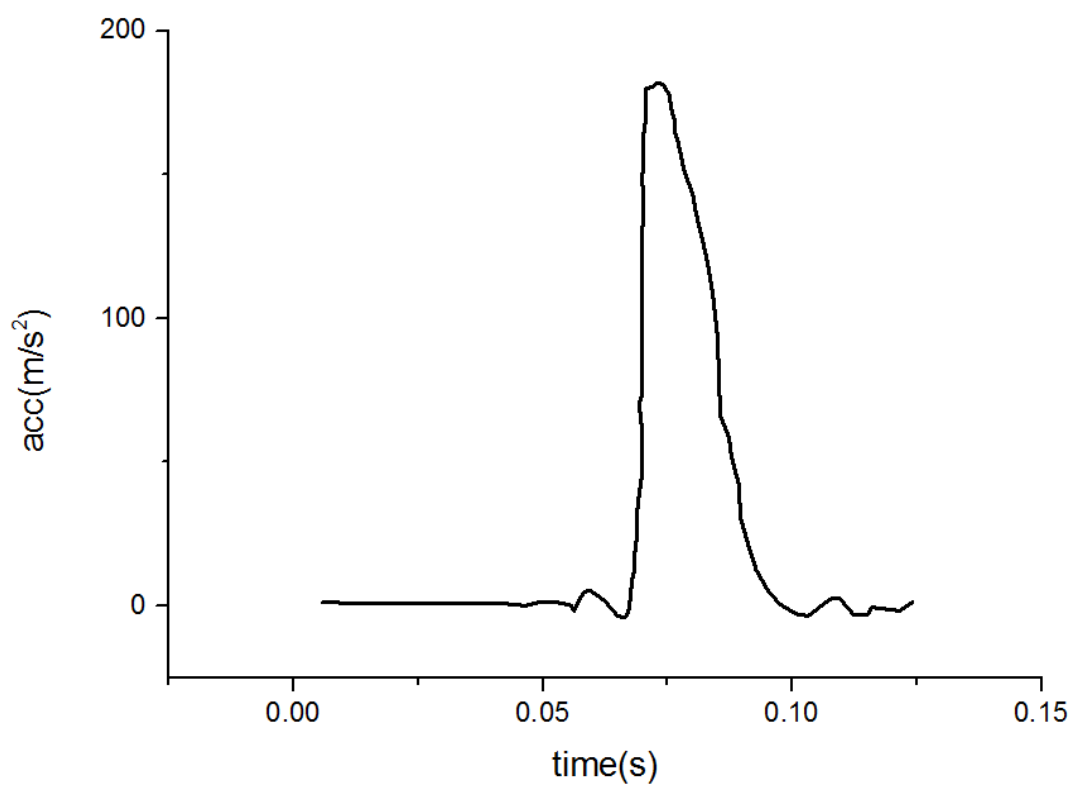

Fig.5. Acceleration curve at the center of the bushing

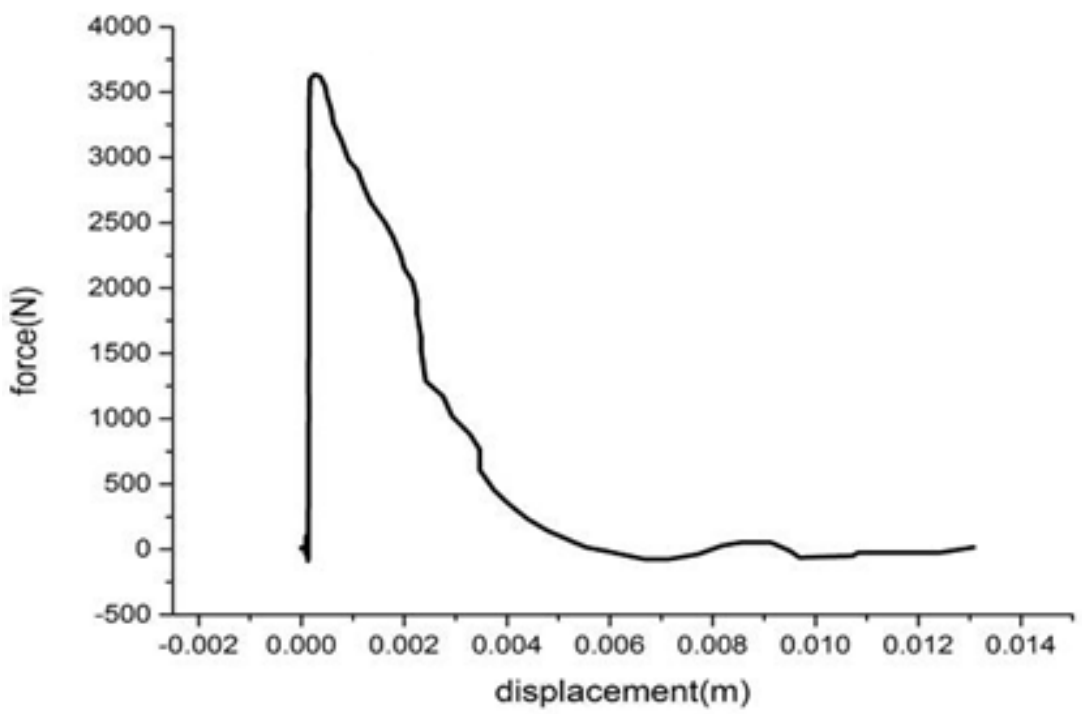

Fig.6. Rubber bushing impact characteristic curve 


\section{Modeling and Simulation on the Rubber Bushing}

Using solid mesh to model the rubber bushing, material properties are assigned to the Yeoh model of rubber material under the condition of large deformation, the model is imported into HyperWorks/Ls-Dyna to calculate, then the solid modeling of the rubber bushing in accordance with the prescribed impact velocity method of benchmarking, to verify the feasibility of the solid mesh model. Finally, the solid mesh model is imported into the simulation of the whole vehicle, in order to determine whether the method is helpful to improve the accuracy of vehicle crash simulation.

\section{Benchmarking Analysis of Bushing}

The acceleration in the inner loop of the simulation model was output, compared with the prescribed impact velocity method acceleration values as shown in Figure 7.

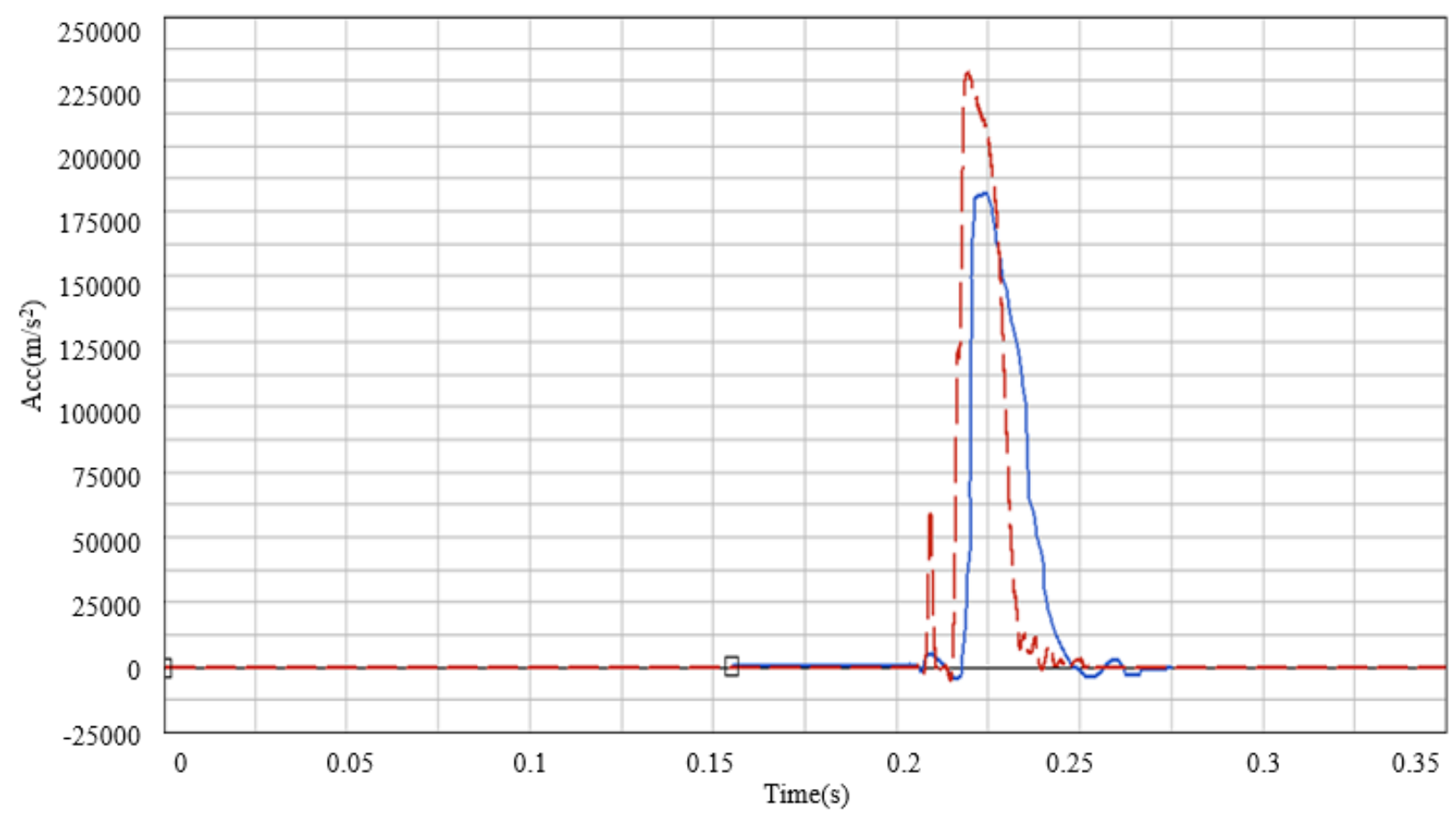

Fig.7. Comparison of test and simulation acceleration

Comparison shows that the two curves are consistent, but the difference of the peak value is $21.6 \%$, the difference of the pulse width is $17.1 \%$, accuracy of simulation benchmarking is acceptable.

The hourglass of the calculation model can be controlled within a reasonable range, in the simulation calculation, there is no negative volume and node speed is not controllable. Based on the above analysis, it is feasible to use the solid modeling method for vehicle crash simulation.

\section{Comparison of Modeling Methods in Vehicle Crash Simulation}

The rubber bushing is modeled by the traditional star three spring connection mode in the whole vehicle simulation model, spring stiffness is 1.6 times of the static stiffness of the rubber. The vehicle model was set up by HyperMesh and exported to $\mathrm{K}$ file.

The rubber bushing is modeled by solid mesh modeling, and exported to $\mathrm{K}$ file. $\mathrm{K}$ files used different modeling method; the other calculation parameters are the same.

Two K files were calculated using Ls-Dyna. Solid model computing time is $7.2 \%$ more than the star three spring model. The acceleration curve of the lower end of the B -pillar is compared with the acceleration curve of the real vehicle collision as shown in Figure 8. 


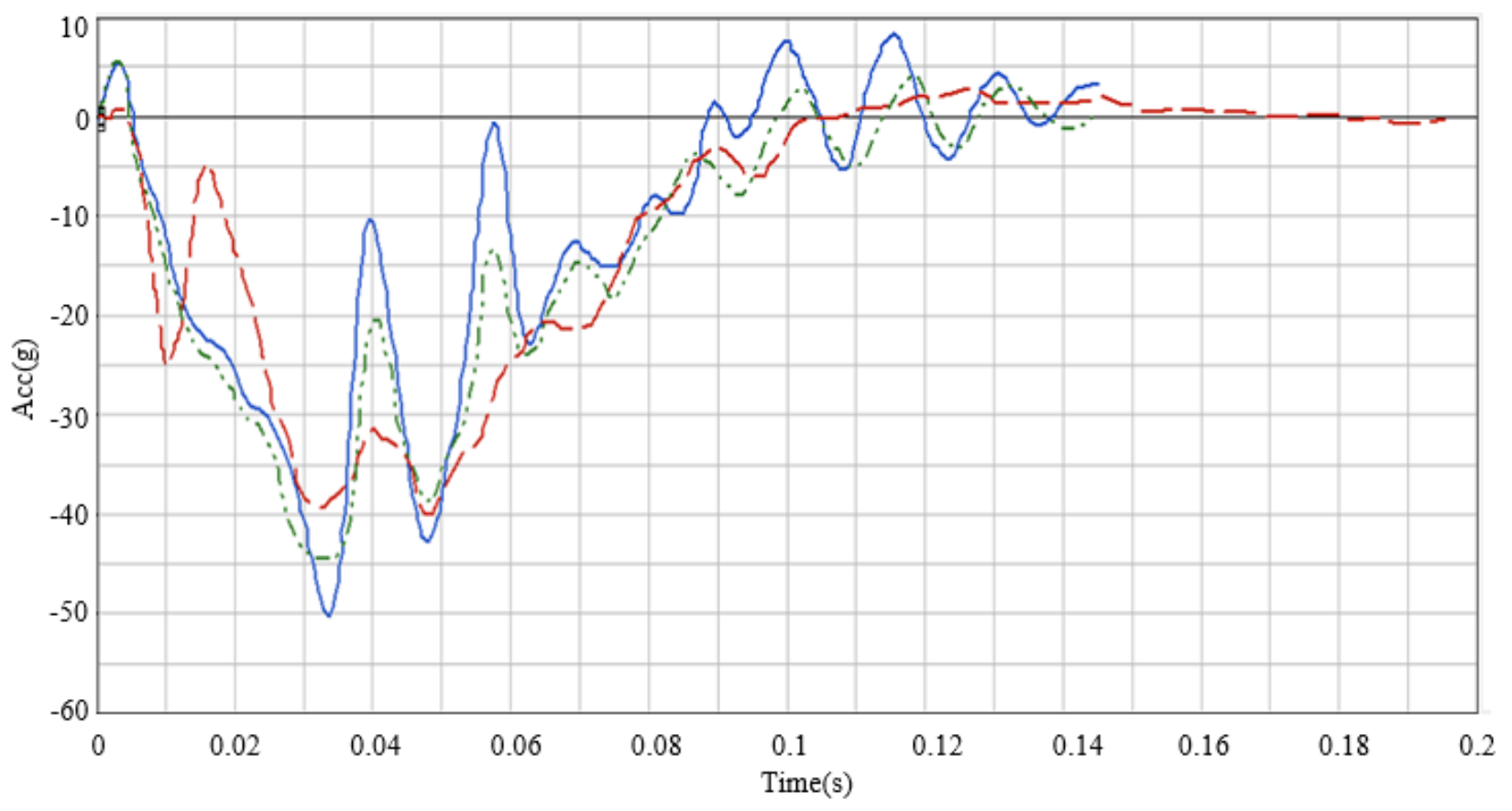

Fig.8. Comparison between two modeling methods and real vehicle test

The modeling methods of solid element and the star three spring model are in line with the trend of the wave form, only the second peak are lower. The solid element model is more close to the real vehicle crash acceleration curve than the star three spring model. The simulation results show that, the difference of acceleration amplitude peak value is $15.38 \%$ and $28.02 \%$ respectively.

The hourglass energy of the solid element. Vehicle kinetic energy is gradually converted into internal energy, the total energy change is about $1.67 \%$, the hourglass energy is about $2.33 \mathrm{~kJ}$, accounting for $1.9 \%$ of the total energy.

The hourglass in the calculation can be within the acceptable range, it shows that it is feasible to model the rubber bushing for solid element.

\section{Summary}

(1) The bushing is modeled by the solid element, material properties are assigned to the Yeoh model. The impact characteristic of the rubber bushing is basically consistent with the test results in simulation analysis of the benchmarking method. It shows that it is feasible to use the Yeoh model to predict the large deformation response of rubber bushing under impact load.

(2) The solid element model was substituted into the vehicle finite element simulation, the acceleration history at the lower end of the B -pillar was collected, the simulation results of the star three spring connection and the result of the whole vehicle test are compared and analyzed. The study found that the use of solid element for rubber modeling can remarkable improve the simulation accuracy of vehicle collision, and the hourglass can be controlled at a reasonable level, and no negative volume.

The paper has carried on the test and the simulation contrast analysis to the rubber bushing to a certain extent. However, due to the precision of the lifting equipment and the guide rail is not enough, impact test data has interference factors, impact testing machine needs to be further improved. The improved method is the linear axis guide rail for guiding the hammer, the test needs further improvement and perfection. 


\section{Reference:}

[1] Jinhuan Zhang, Huiliang Du, Chunsheng Ma. Automobile crash safety design [M]. Peking: Tsinghua university press, 2010:87-132. "In Chinese”

[2] Yang L, Shim V, Lim C. A visco-hyperelastic approach to modelling the constitutive behaviour of rubber [J]. International Journal of Impact Engineering, 2000, 24(6-7):545-560.

[3] Rendek M, Lion A. Amplitude dependence of filler-reinforced rubber: Experiments, constitutive modelling and FEM - Implementation [J]. International Journal of Solids and Structures, 2010, 47(21):2918-2936.

[4] Zhang J, Richards C. Parameter identification of analytical and experimental rubber isolators represented by Maxwell models [J]. Mechanical Systems and Signal Processing, 2007, 21(7):2814-2832.

[5] Li J, Mayau D, Song F. A constitutive model for cavitation and cavity growth in rubber-like materials under arbitrary tri-axial loading [J]. International Journal of Solids and Structures, 2007, 44(18-19):6080-6100.

[6] Xia Y, Zhou Q, Wang P, et al. Development of high-efficiency modeling technique for weld-bonded steel joints in vehicle structures-Part I: Static experiments and simulations [J]. International Journal of Adhesion and Adhesives, 2009, 29(4):414-426.

[7] Xia Y, Zhou Q, Wang P, et al. Development of a high-efficiency modeling technique for weld-bonded steel joints in vehicle structures, Part II: Dynamic experiments and simulations [J]. International Journal of Adhesion and Adhesives, 2009, 29(4):427-433.

[8] Qishuai Gao. Design and impact test analysis of impact test bech for automobile parts [D]. Harbin Institute of Technology, 2008:13-25. "In Chinese” 